\title{
Rendimento e conservação pós-colheita de bulbos de cebola com doses de nitrogênio e potássio
}

\author{
Geraldo M de Resende; Nivaldo Duarte Costa; José Maria Pinto \\ Embrapa Semi-Árido, C. Postal 23, 56302-970 Petrolina-PE; gmilanez@ cpatsa.embrapa.br
}

\section{RESUMO}

O experimento foi conduzido de março a novembro de 2001, em Petrolina-PE, com o objetivo de avaliar a influência de doses de nitrogênio e potássio aplicadas via fertirrigação sobre o rendimento e conservação pós-colheita da cebola. Utilizou-se a cultivar Franciscana IPA-10, no delineamento experimental de blocos ao acaso, em esquema fatorial $4 \times 3$, compreendendo quatro doses de nitrogênio $(0 ; 60$; 120 e $\left.180 \mathrm{~kg} \mathrm{ha}^{-1}\right)$ e três doses de potássio $\left(0 ; 90\right.$ e $180 \mathrm{~kg} \mathrm{ha}^{-1} \mathrm{de}$ $\mathrm{K}_{2} \mathrm{O}$ ), com três repetições. A adubação nitrogenada apresentou efeitos lineares positivos na produtividade comercial independente da adubação potássica. Para a produtividade não comercial (refugos) constataram-se reduções lineares na ausência da adubação potássica e nas doses de 90 e $180 \mathrm{~kg} \mathrm{ha}^{-1}$ de $\mathrm{K}_{2} \mathrm{O}$, com o incremento das doses de nitrogênio. $\mathrm{O}$ aumento das doses de nitrogênio proporcionou um incremento linear na massa fresca dos bulbos. Não se constataram efeitos significativos para perda de massa aos 40 e 80 dias após cura, com a aplicação de nitrogênio e potássio.

Palavras-chave: Allium cepa, cultivo de inverno, produtividade, adubação NK.

\begin{abstract}
Yield and post-harvest conservation of onion bulbs using doses of nitrogen and potassium

This study was carried out from March to November 2001, in Petrolina, Pernambuco State, Brazil, to evaluate the influence of nitrogen and potassium levels applied through fertirrigation, on yield and post-harvest quality of onion bulbs. Cultivar Franciscana IPA10 was used in a randomized complete block design, in a 4 x 3 factorial arrangement, with four nitrogen levels $(0 ; 60 ; 120$ and 180 $\left.\mathrm{kg} \mathrm{ha}^{-1}\right)$ and three potassium levels $\left(0 ; 90\right.$ and $180 \mathrm{~kg} \mathrm{ha}^{-1}$ of $\left.\mathrm{K}_{2} \mathrm{O}\right)$ with three replications. The applied levels of nitrogen showed linear effects on the commercial yield independent of the potassium fertilization. The non-commercial yield (calls) showed linear reductions in the three potassium levels, with the increase in nitrogen levels. With the increase of applied nitrogen levels a linear effect on the fresh mass of the bulbs was observed. There were no significant effects of nitrogen and potassium application on the mass loss of the bulbs at 40 and 80 days after cure.
\end{abstract}

Keywords: Allium cepa, yield, winter cultivation, NK fertilization.

(Recebido para publicação em 10 de dezembro de 2007; aceito em 13 de maio de 2009)

(Received in December 10, 2007; accepted in May 13, 2009)

$\mathrm{N}_{\mathrm{h}}^{\circ}$ Brasil, a cebola ocupa entre as hortaliças o terceiro lugar em importância econômica (Souza \& Resende, 2002). A fertilização constitui uma das práticas agrícolas mais caras e de maior retorno econômico, resultando em maiores rendimentos e em produtos mais uniformes e de maior valor comercial (Ricci et al., 1995). O fato relevante de se determinar uma dose adequada às culturas relaciona-se às perdas por volatilização e lixiviação que ocorrem com nitrogênio e potássio, que segundo Pôrto et al. (2007) são os mais absorvidos em termos de porcentagem na matéria seca da cebola. $\mathrm{O}$ diâmetro do bulbo e a produtividade aumentaram com o incremento das doses até $80 \mathrm{~kg}$ $\mathrm{ha}^{-1}$ de $\mathrm{N}$, não havendo diferenças significativas da dose de $120 \mathrm{~kg} \mathrm{ha}^{-1}$ de $\mathrm{N}$ (Singh \& Sharma, 1991). Faria \& Pereira. (1992), verificaram nas condições do
Vale do São Francisco, que o nível econômico de $\mathrm{N}$ para produtividade na cultura da cebola é de $115 \mathrm{~kg} \mathrm{ha}^{-1}$. Vachchani \& Patel (1996) aplicando 150 $\mathrm{kg} \mathrm{ha}^{-1}$ de $\mathrm{N}$ na cultura da cebola obtiveram aumento na altura das plantas, número de folhas por planta, peso e tamanho do bulbo e na produtividade, porém sem mostrar diferenças significativas na dose de $100 \mathrm{~kg} \mathrm{ha}^{-1}$ de $\mathrm{N}$, para o peso do bulbo e a produtividade. Aumentos na produção de cebola com aplicações de doses de $\mathrm{N}$ têm sido relatadas por diversos autores (Dixit, 1997; Hussaini et al., 2000; Neeraja et al., 2001; Diaz-Perez et al., 2003; Singh et al., 2000; Boyhan et al., 2007). Entretanto, outros trabalhos não encontraram respostas positivas à aplicação de nitrogênio (Patel et al., 1992; Batal et al., 1994; Hensel \& Shumaker, 1991).
Apesar das plantas de cebola extrairem grandes quantidades de potássio, respostas da cultura a esse nutriente, de maneira geral, não têm sido observadas (Filgueira, 2003) e há poucos resultados que confirmam efeito significativo do fertilizante potássico na produtividade, embora não se conheça o seu efeito sobre a qualidade e a conservação do produto (Magalhães, 1993). Sharma (1992) e Shukla et al. (1992), obtiveram um incremento na produtividade da cultura com a aplicação de 40 kg/ ha de potássio, sem verificar qualquer efeito adicional quando se aplicaram doses superiores $\left(80\right.$ e $160 \mathrm{~kg} \mathrm{ha}^{-1}$ de $\mathrm{K}_{2} \mathrm{O}$ ).

No que se refere a bulbos armazenados sob condições ambientais, Singh \& Dhankhar (1991) verificaram que a perda de peso e a incidência de bulbos podres e brotados aumentaram com o incremen- 




Figura 1. Produtividade comercial de bulbos de cebola nas doses $0 ; 90$ e $180 \mathrm{~kg} \mathrm{ha}^{-1} \mathrm{de}$ potássio em função das doses de nitrogênio (Commercial yield of onion bulbs on the levels 0 ; 90 and $180 \mathrm{~kg} \mathrm{ha}^{-1}$ at potassium in response to the nitrogen levels). Petrolina, Embrapa SemiÁrido, 2001.

to das doses de nitrogênio (80 a $160 \mathrm{~kg}$ $\mathrm{ha}^{-1} \mathrm{de} \mathrm{N}$ ) e reduziu com a dose de $100 \mathrm{~kg}$ $\mathrm{ha}^{-1}$ de $\mathrm{K}_{2} \mathrm{O}$. As melhores respostas para a qualidade de armazenamento foram obtidas com as doses de $80 \mathrm{~kg} \mathrm{ha}^{-1}$ de $\mathrm{N}$ mais $100 \mathrm{~kg} \mathrm{ha}^{-1}$ de $\mathrm{K}_{2} \mathrm{O}$.

Neste trabalho, avaliou-se o efeito de doses de nitrogênio e potássio na produtividade e conservação pós-colheita de bulbos de cebola no Vale do São Francisco, em condições de inverno.

\section{MATERIAL E MÉTODOS}

O experimento foi conduzido de março a novembro de 2001, em campo experimental da Embrapa Semi-Árido, em Petrolina-PE. O solo classificado como Latossolo Vermelho Amarelo Distróferrico (Embrapa, 1999), apresentou $\mathrm{pH}\left(\mathrm{H}_{2} \mathrm{O}\right)=$ 6,$8 ; \mathrm{Ca}=2,1 \mathrm{cmol} \mathrm{dm}^{-3} ; \mathrm{Mg}=0,7 \mathrm{cmol} \mathrm{dm}^{-3}$ $\mathrm{Na}=0,01 \mathrm{cmol}_{\mathrm{c}} \mathrm{dm}^{-3} ; \mathrm{K}=0,27 \mathrm{cmol} \mathrm{dm}_{\mathrm{c}}^{-3}$; $\mathrm{Al}=0,00 \mathrm{cmol} \mathrm{dm}^{-3}, \mathrm{P}($ Mehlich $)=32,0 \mathrm{mg}$ $\mathrm{dm}^{-3} \mathrm{e}$ M.O. $=6,8 \mathrm{~g} \mathrm{~kg}^{-1}$.

O delineamento experimental utilizado foi blocos ao acaso, em esquema fatorial $4 \times 3$, compreendendo quatro doses de nitrogênio $\left(0 ; 60 ; 120\right.$ e $\left.180 \mathrm{~kg} \mathrm{ha}^{-1}\right)$ e três doses de potássio (0; 90 e $180 \mathrm{~kg}$ $\mathrm{ha}^{-1}$ ) com três repetições. As unidades experimentais foram distanciadas uma da outra por 2,0 m e constituiram-se de um canteiro com oito linhas de 3,0 m de comprimento, espaçadas de $0,15 \mathrm{~m}$, com 0,10 $\mathrm{m}$ entre plantas, perfazendo uma área total de $3,6 \mathrm{~m}^{2}(3,0 \times 1,2 \mathrm{~m})$, sendo utilizadas como área útil as seis linhas cen- trais, retirando-se $0,50 \mathrm{~m}$ em cada extremidade $\left(1,80 \mathrm{~m}^{2}\right)$.

A adubação de plantio constou de $60 \mathrm{~kg} \mathrm{ha}^{-1}$ de $\mathrm{P}_{2} \mathrm{O}_{5}$ (superfosfato simples) baseada na análise do solo. As adubações nitrogenada (uréia) e potássica (sulfato de potássio) foram aplicadas via água de irrigação, diariamente, utilizando-se injetores hidráulicos de fertilizantes, de acordo com os diferentes tratamentos, iniciando-se aos cinco dias após o transplantio, sendo aplicadas por 44 vezes para o nitrogênio, totalizando sessenta dias consecutivos e por 59 vezes para o potássio totalizando setenta dias

A semeadura da cultivar Franciscana IPA-10 foi feita em 22 de março de 2001 e o transplante efetuado 30 dias após. O preparo do solo constou de aração, gradagem e levantamento dos canteiros a 0,20 m de altura. A cultura foi mantida no limpo através de capinas manuais e a irrigação por microaspersão realizada diariamente, com lâminas em torno de 5 $\mathrm{mm}$, baseada na evaporação do tanque classe A, e suspensa 20 dias antes da colheita. Os tratos fitossanitários foram realizados de acordo com as recomendações para a cultura da cebola.

A colheita foi realizada em 14 de agosto de 2001 quando as plantas apresentaram sinais avançados de senescência, como amarelecimento e seca das folhas e mais de $70 \%$ das plantas encontravam-se estaladas. A cura foi realizada ao sol por 3 dias e 12 dias à sombra em galpão ventilado. consecutivos.
Foram avaliadas a produtividade comercial de bulbos (bulbos perfeitos e com diâmetro transversal acima de $35 \mathrm{~mm}$ ) e não comerciais (refugos) (com diâmetro inferior a $35 \mathrm{~mm}$ ) expressas em $\mathrm{t} \mathrm{ha}^{-1}$, aos 15 dias após a cura. A massa fresca de bulbo ( $\mathrm{g} \mathrm{bulbo}^{-1}$ ) foi determinada dividindo-se o peso de bulbos comerciais após a cura pelo número de bulbos. Após o período de cura, os bulbos foram armazenados à temperatura ambiente (média mensal de $26,1^{\circ} \mathrm{C}$ ) e umidade relativa média $(57,3 \%)$ e realizadas pesagens aos 40 e 80 dias, sendo os valores comparados àqueles obtidos ao final da cura (15 dias após colheita). Os valores foram transformados em porcentagem de perda de massa. A classificação de bulbos comerciais segundo o diâmetro transversal ( $\mathrm{mm}$ ) foi feita de acordo com Brasil (1995) em classe 2 (>35 até $50 \mathrm{~mm}$ de diâmetro); classe 3 (>50 até $70 \mathrm{~mm})$; classe 4 (>70 ate $90 \mathrm{~mm}$ ) e classe 5 (>90 $\mathrm{mm})$. Os dados foram expressos em porcentagem. Os dados coletados foram submetidos à análise de variância e regressão polinomial ao nível de $5 \%$ de probabilidade. Os dados de porcentagem foram transformados em arcoseno $\sqrt{P / 100}$ para efeitos de análise.

\section{RESULTADOS E DISCUSSÃO}

Observou-se efeitos significativos para as doses de nitrogênio e potássio, assim como para sua interação, variando com as características avaliadas.

As doses de nitrogênio apresentaram efeitos significativos lineares positivos na produtividade comercial na ausência da adubação potássica, como nas doses de 90 e $180 \mathrm{~kg} \mathrm{ha}^{-1}$ de $\mathrm{K}_{2} \mathrm{O}$ (Figura 1). Observa-se, ainda pela Figura 1 incremento na produtividade comercial com o aumento também das doses de potássio, contudo, em baixa magnitude, apresentando as doses de 90 e $180 \mathrm{~kg}$ $\mathrm{ha}^{-1}$ de $\mathrm{K}_{2} \mathrm{O}$ produtividades similares. Neste contexto, infere-se ser a dose de $90 \mathrm{~kg} \mathrm{ha}^{-1}$ de $\mathrm{K}_{2} \mathrm{O}$ a mais adequada nas condições estudadas, tendo em vista que na maior dose de $\mathrm{N}\left(180 \mathrm{~kg} \mathrm{ha}^{-1}\right)$ apresentou 58,7 t ha-1 contra 58,8 $\mathrm{t} \mathrm{ha}^{-1}$, obtidos pela dose de $180 \mathrm{~kg} \mathrm{ha}^{-1}$ de $\mathrm{K}_{2} \mathrm{O}$. Pelos resultados obtidos infere-se ser 
as doses de $180 \mathrm{~kg} \mathrm{ha}^{-1}$ de nitrogênio e $90 \mathrm{~kg} \mathrm{ha}^{-1}$ de $\mathrm{K}_{2} \mathrm{O}$ as mais adequadas em termos produtivos. Respostas positivas da aplicação de nitrogênio na cultura da cebola têm sido relatadas por diversos autores até as doses de $150 \mathrm{~kg} \mathrm{ha}^{-1}$ (DiazPerez et al., 2003; Singh et al., 2004) e $200 \mathrm{~kg} \mathrm{ha}^{-1}$ (Neeraja et al., 2001) de forma isolada. Em interação, Singh et al. (2000) verificaram maiores produtividades com $100 \mathrm{~kg} / \mathrm{ha}$ de $\mathrm{N}$ e $83 \mathrm{~kg} \mathrm{ha}^{-1}$ de $\mathrm{K}_{2} \mathrm{O}$; Syed et al. (2000) com $120 \mathrm{~kg} / \mathrm{ha}$ de $\mathrm{N}$ e $90 \mathrm{~kg} \mathrm{ha}^{-1}$ de $\mathrm{K}_{2} \mathrm{O}$; enquanto Mandira \& Khan (2003), obtiveram melhores rendimentos com $150 \mathrm{~kg} \mathrm{ha}^{-1} \mathrm{de}$ $\mathrm{N}$ e $75 \mathrm{~kg} \mathrm{ha}^{-1}$ de $\mathrm{K}_{2} \mathrm{O}$.

A literatura relata um antagonismo entre estes elementos; no entanto, os resultados obtidos sugerem uma resposta complementar, todavia, evidenciando ser o nitrogênio o principal fator que afeta positivamente a produtividade. De acordo com Silva Junior. (1987) este antagonismo tem origem em adubações desequilibradas, afirmação corroborada por Resende et al. (1997), que afirmam que há um efeito significativo e complementar na absorção de nitrogênio e potássio, e que o importante é a necessidade de um adequado nível de potássio para incrementar a produtividade com a adição de nitrogênio.

Resultados similares foram obtidos para a produtividade não comercial (refugos) em relação inversa, onde constatou-se reduções lineares na ausência da adubação potássica, assim como nas doses de 90 e $180 \mathrm{~kg} \mathrm{ha}^{-1}$ de $\mathrm{K}_{2} \mathrm{O}$, com o incremento das doses de nitrogênio (Figura 2). Observa-se que tanto as aplicações de nitrogênio, como as de potássio promoveram menores produções de bulbos considerados refugos, o que denota uma interação entre esses nutrientes já comentada na variável anterior. Assim como se pode verificar que o efeito da aplicação de nitrogênio foi mais expressiva do que a promovida pelo potássio, verificado pela inclinação e variação entre linhas. Cabe salientar que como a característica anterior, houve similaridade no comportamento das doses de 90 e $180 \mathrm{~kg} \mathrm{ha}^{-1}$ de $\mathrm{K}_{2} \mathrm{O}$, sobretudo, na maior dose de nitrogênio. May (2006) encontrou com aplicação de 115 $\mathrm{kg} \mathrm{ha}^{-1}$ de $\mathrm{N}$ e $150 \mathrm{~kg} \mathrm{ha}^{-1}$ de $\mathrm{K}_{2} \mathrm{O}$, reduções na quantidade de bulbos considerados não comerciais (refugos).

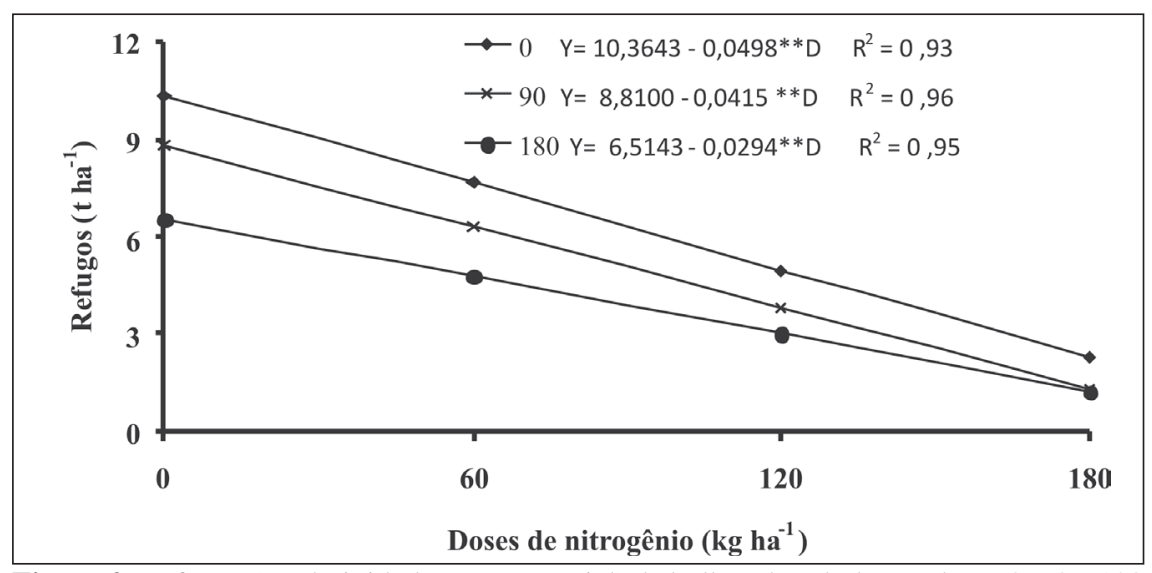

Figura 2. Refugos (produtividade não comercial) de bulbos de cebola nas doses 0; 90 e 180 $\mathrm{kg} / \mathrm{ha}$ de potássio em função das doses de nitrogênio (Non-commercial yield of onion bulbs (culls) on the levels of $0 ; 90$ and $180 \mathrm{~kg} \mathrm{ha}^{-1}$ of potassium in response to the nitrogen levels). Embrapa Semi-Árido, 2001.

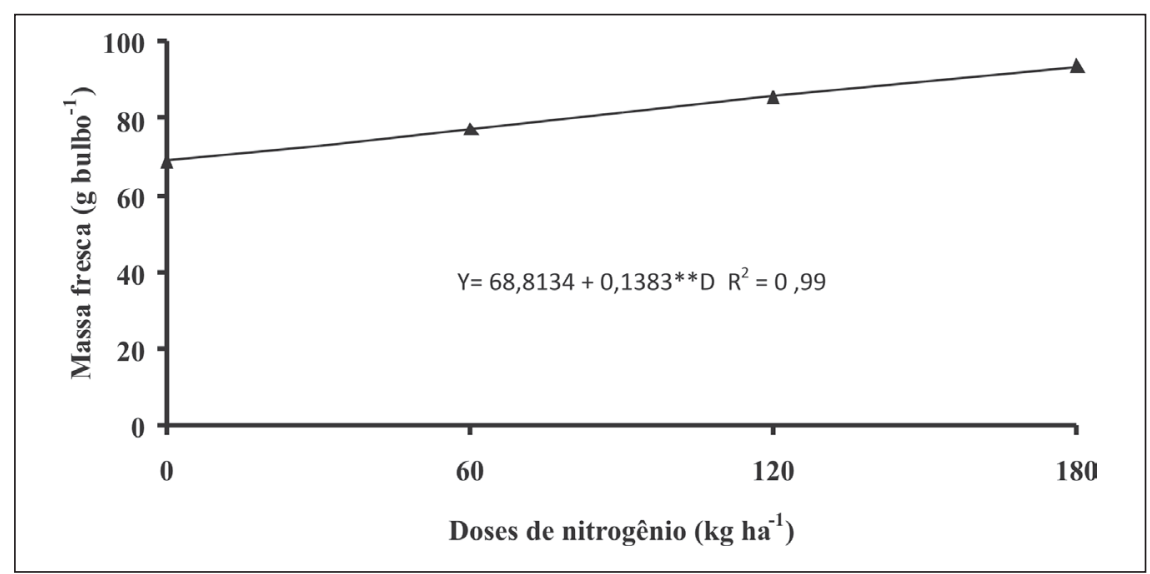

Figura 3. Massa fresca de bulbos de cebola em função das doses de nitrogênio (fresh mass of onion bulbs in response to the nitrogen levels). Petrolina, Embrapa Semi-Árido, 2001.

Para a massa fresca do bulbo verificou-se efeito apenas para doses de nitrogênio (Figura 3). O aumento das doses proporcionou um incremento gradativo na massa fresca dos bulbos. Estes resultados mostram a capacidade de resposta da cebola à aplicação de nitrogênio e alicerça as afirmações de diversos autores que relatam que o elemento contribui marcadamente para produção de bulbos de maior tamanho (massa fresca) e consequientemente melhor produtividade da cultura (Singh \& Sharma, 1991; Faria \& Pereira, 1992; Vachchani \& Patel, 1996; Hussaini et al., 2000).

A classificação de bulbos comerciais de cebola foi influenciada pela interação dos fatores estudados (Tabela 1), sendo obtidos apenas bulbos classes 2 e 3 . No que se refere à classificação de bul- bos classe 2 que são bulbos de tamanho inferior (>35 até $50 \mathrm{~mm}$ de diâmetro), verificou-se tanto na ausência como na presença da adubação potássica reduções lineares na produção de bulbos nesta classe com o incremento das doses de nitrogênio. Resultados similares foram obtidos para classe 3 que são bulbos maiores ( $>50$ até $70 \mathrm{~mm}$ de diâmetro) em ordem inversa, ou seja, obtiveram efeitos lineares positivos na ausência e nas dose de 90 e $180 \mathrm{~kg} \mathrm{ha}^{-1}$ de $\mathrm{K}_{2} \mathrm{O}$, onde registrou-se com o incremento das doses de nitrogênio e potássio maiores percentagens de bulbos de tamanho superior (Tabela 1). Todavia, como as características anteriores, apesar das aplicações de potássio mostrarem-se significativas, foram de baixa magnitude. Estes resultados corroboram as afirmações de Maier et al. (1990), que rela- 
Tabela 1. Equações de regressão para classificação, em classes (\%), segundo o diâmetro transversal de bulbos de cebola de doses (D) de potássio $(\mathrm{K})$ e nitrogênio $(\mathrm{N})$ (regression equations for classification (\%), according to the transversal diameter of onion bulbs to the potassium (K) and nitrogen (N) levels (D). Petrolina, Embrapa Semi-Árido, 2001.

\begin{tabular}{llll}
\hline Características & \multicolumn{3}{c}{ Equações de regressão } \\
\hline \multirow{3}{*}{ Classe 2 } & $\mathrm{N}: \mathrm{K}(0)$ & $\mathrm{Y}=37,0901-0,0897^{*} \mathrm{D}$ & $\mathrm{R}^{2}=0,70$ \\
& $\mathrm{~N}: \mathrm{K}(90)$ & $\mathrm{Y}=34,8972-0,0641^{* *} \mathrm{D}$ & $\mathrm{R}^{2}=0,70$ \\
& $\mathrm{~N}: \mathrm{K}(180) \mathrm{Y}=30,1328-0,1050^{\star *} \mathrm{D}$ & $\mathrm{R}^{2}=0,85$ \\
\hline \multirow{3}{*}{ Classe 3 } & $\mathrm{N}: \mathrm{K}(0)$ & $\mathrm{Y}=52,9098+0,0897^{\star \star} \mathrm{D}$ & $\mathrm{R}^{2}=0,78$ \\
& $\mathrm{~N}: \mathrm{K}(90)$ & $\mathrm{Y}=55,1027+0,0641^{* \star} \mathrm{D}$ & $\mathrm{R}^{2}=0,72$ \\
& $\mathrm{~N}: \mathrm{K}(180) \mathrm{Y}=59,8671+0,0647^{\star *} \mathrm{D}$ & $\mathrm{R}^{2}=0,85$ \\
\hline
\end{tabular}

****Significativo ao nível de 5 e $1 \%$ de probabilidade, pelo teste de $\mathrm{F}$.

tam ser o nitrogênio de grande importância para o aumento de bulbos comercialmente desejáveis, dos quais, segundo Silva et al. (1991), os de classe 3 têm a preferência do mercado consumidor nacional.

Para perda de massa, não se constatou efeitos significativos aos 40 dias, verificando-se variações para doses de nitrogênio entre 4,2 e 7,1\% e 5,5 a 6,3\% para doses de potássio. Da mesma forma, aos 80 dias após a cura, observaram-se variações para doses de nitrogênio entre 14,5 e $14,7 \%$ e 13,1 a $15,0 \%$ para doses de potássio. Apesar dos resultados não serem significativos, evidenciou-se que as perdas foram maiores com o aumento das doses de nitrogênio e menores com o incremento das doses de potássio. Singh \& Dhankhar (1991) verificaram em bulbos armazenados sob condições ambientais que a perda de massa e a incidência de bulbos podres e brotados aumentaram com o incremento das doses de nitrogênio (80 a $160 \mathrm{~kg} \mathrm{ha}^{-1}$ de $\mathrm{N}$ ) e reduziram com a dose de $100 \mathrm{~kg} \mathrm{ha}^{-1}$ de $\mathrm{K}_{2} \mathrm{O}$. As doses de $80 \mathrm{~kg} \mathrm{ha}^{-1}$ de $\mathrm{N}$ mais $100 \mathrm{~kg} \mathrm{ha}^{-1}$ de $\mathrm{K}_{2} \mathrm{O}$ obtiveram as melhores respostas em termos de armazenamento.

Vale a pena salientar que a área utilizada para o experimento apresentou solo com teor de $\mathrm{K}=0,27 \mathrm{cmol}_{\mathrm{c}} \mathrm{dm}^{-3}$, o que é considerado alto e que de acordo com Cavalcanti (1998) necessitaria de incorporação de $90 \mathrm{~kg} \mathrm{ha}^{-1}$ de $\mathrm{K}_{2} \mathrm{O}$. Os resultados obtidos indicaram ser esta a dose mais adequada quando associada (em interação) ao nitrogênio, que proporciona maior produtividade comercial, menores produções de bulbos não comerciais (refugos) e bulbos de maior diâmetro. No entanto, caso o presente estudo fosse realizado em um solo com teores menores de potássio, certamente se obteria resultados provavelmente superiores.

Os resultados obtidos permitem concluir que as maiores produtividades comerciais de bulbos de cebola foram obtidas com as doses de $180 \mathrm{~kg} \mathrm{ha}^{-1}$ de nitrogênio e $90 \mathrm{~kg} \mathrm{ha}^{-1}$ de $\mathrm{K}_{2} \mathrm{O}$; que as aplicações de nitrogênio e potássio promoveram redução na produtividade não comercial de bulbos (refugos) e ainda que, na interação o nitrogênio é o nutriente de maior importância, em termos de produtividade e diâmetro do bulbo, sendo potássio mais relevante na conservação pós-colheita.

\section{REFERÊNCIAS}

BATAL KM, BONDARI K; GRANBERRY DM; MULLINIX BG. 1994. Effects of source, rate, and frequency of $\mathrm{N}$ application on yield, marketable and rot incidence of sweet onion (Allium cepa L. cv. Granex33). Journal of Horticultural Science 69: 1043-1051.

BOYHAN GE; TORRANCE RL; HILL CR. 2007. Effects of nitrogen, phosphorus, and potassium rates and fertilizer sources on yield and leaf nutrient status of short-day onions. HortScience 42: 429-748.

BRASIL. Ministério da Agricultura, Abastecimento e Reforma Agrária. 1995. Portaria n. 529 de 18 agosto 1995. Diário Oficial da República Federativa do Brasil, Brasília, Seção1. p.13513.

CAVALCANTI FJA. (Coord.). 1998. Recomendações de adubação para o Estado de Pernambuco: 2a aproximação. Recife: IPA. 198p.

DIAZ-PEREZ JC; PURVIS AC; PAULK JT. Bolting, yield, and bulb decay of sweet onion as affected by nitrogen fertilization. 2003. Journal of the American for Society Horticultural Science 128: 144-149.

DIXIT SP. Response of onion (Allium cepa L.) to nitrogen and farmyard manure in dry temperate high hills of Himachal Pradesh. 1997. Indian Journal of Agricultural Sciences 67: 222-223.
EMBRAPA. 1999. Sistema Brasileiro de Classificação de Solos. Brasília: Embrapa Produção de Informações (SPI). 412 p.

FARIA CM; PEREIRA JR. 1992. Fontes e níveis de nitrogênio na produtividade da cebola no Vale do São Francisco. Pesquisa Agropecuária Brasileira 27: 403-407.

FILGUEIRA FAR. 2003. Novo manual de olericultura: agrotecnologia moderna produção e comercialização de hortaliças. 2. ed., Viçosa: Editora UFV. 412p.

HENSEL DR; SHUMAKER JR. 1991. Plant bed configuration, fertilization rate and application method, and cultivar effects on sweet onion production. Proceedings of the Florida State Horticultural Society 133: 105-107.

HUSSAINI MA; AMANS EB, RAMALAN AA. 2000. Yield, bulb size distribution, and storability of onion (Allium cepa L.) under different levels of $\mathrm{N}$ fertilization and irrigation regime. Tropical Agriculture 77: 145-149.

MAGALHÃES JR. 1993. Nutrição e adubação da cebola. In: SIMPOSIO SOBRE NUTRIÇÃO E ADUBAÇÃO DE HORTALIÇAS, 1990, Jaboticabal, SP. Nutrição e adubação de hortaliças: Anais... Piracicaba: POTAFOS. p. 381-399.

MAIER NA; DAHLENBURG AP; TWIGDEN T K. 1990. Effect of nitrogen on the yield and quality of irrigated onions (Allium cepa L.) cv. Cream Gold grown on siliceous sands. Australian Journal of Experimental Agriculture 30: 845-851.

MANDIRA C; KHAN AH. 2003. Effect of nitrogen and potassium on growth, yield and yield attributes of onion. New Agriculturist 14: 9-11.

MAY A. 2006. Desempenho de híbridos de cebola em função da população de plantas e fertilização nitrogenada $e$ potássica. Jaboticabal: UNESP-FCAV, 142p. (Tese doutorado).

NEERAJA G; REDDY KM; REDDY MS; RAO VP. 2001. Influence of irrigation and nitrogen levels on bulb yield, nutrient uptake an nitrogen use efficiencies in rabi onion (Allium cepa). Indian Journal of Agricultural Sciences 7: 109-11.

PATEL KP; PATEL BS; SADARIA SG. 1992. Yield and nutrient uptake by onion (Allium cepa L.) as influenced by irrigation, nitrogen and phosphorus. Indian Journal of Agronomy 37: 395-396.

PÔRTO DRQ; CECILIO FILHO AB; MAY A; VARGAS PF. 2007. Acúmulo de macronutrientes pela cultivar de cebola "Superex" estabelecida por semeadura direta. Ciência Rural 37: 949-955.

RESENDE GM; SILVA GL; PAIVA LE; DIAS PF; CARVALHO JG. 1997. Resposta do milho (Zea mays L.) a doses de nitrogênio e potássio em solo da região de Lavras-MG. II. Macronutrientes na parte aérea. Ciência e Agrotecnologia 21: 477-483.

RICCI MSF; CASALI VWD; CARDOSO AA; RUIZ HA. 1995. Teores de nutrientes em duas cultivares de alface adubadas com composto orgânico. Pesquisa Agropecuária Brasileira 30: 1035-1039. 
SHARMA RP. 1992. Effect of planting material, nitrogen and potash on bulb yield of rainy-season onion (Allium cepa). Indian Journal of Agronomy 37: 868-869.

SHUKLA V; RAO KPGK; PRABHAKAR BS. 1992. Effect of nitrogen on bulb yield and keeping quality of onion cultivars. Progressive Horticulture 21: 244-245.

SILVA E; TEIXEIRA LAJ; AMADO TJC. 1991. The increase in onion production in Santa Catarina State, South Brazil. Onion Newsletter for the Tropics 3: 7-9.

SILVA JUNIOR AA. 1987. Adubação mineral e orgânica em repolho. II. Concentração de nutrientes na folha e precocidade. Horticultura Brasileira 5: 15-17.

SINGH D; SHARMA RP. 1991. Effect of soil moisture regimes and nitrogen fertilization on onion. Indian Journal of Agronomy 36: 125-126.
SINGH J; DHANKHAR BS. 1991. Effect of nitrogen, potash and zinc on storage loss of onion bulbs (Allium cepa L.). Vegetable Science 18: 16-23.

SINGH RP; JAIN NK; POONIA BL. 2000. Response of Kharif onion to nitrogen, phosphorus and potash in eastern plains of Rajasthan. Indian Journal of Agricultural Science 70: 871- 872.

SINGH S; YADAV PK; SINGH B. 2004. Effect of nitrogen and potassium on growth and yield of onion (Allium cepa L.) cv. Pusa Red. Haryana Journal of Horticultural Sciences 33: 308-309.
SOUZA RJ, RESENDE GM. 2002. Cultura da cebola. Lavras: UFLA. 115p. (Textos Acadêmicos - Olericultura, 21).

SYED N; MUNIR M; ALIZAI AA; GHAFOOR A. 2000. Onion yield and yield components as function of the levels of nitrogen and potassium application. Pakistan Journal of Biological Sciences 3: 2069-2071.

VACHCHANI MU; PATEL ZG. 1996. Growth and yield of onion (Allium cepa L.) as influenced by levels of nitrogen, phosphorus and potash under south Gujarat conditions. Progressive Horticulture 25: 166-167. 